without reservations. For example, it is by no means certain what proportion of mRNAs contain poly(A). In animals only the histone mRNAs have been shown to lack poly(A) but others may be added to this list and it is too early to assume that all plant mRNAs contain poly(A). It is also probably true that some nuclear RNA molecules that contain poly(A) do not function as mRNAs.

The most favourable situations for the isolation of a single mRNA exist where cells devote a large part of their protein synthesis to the production of one or a small number of specific proteins. One can anticipate that already in a number of laboratories there is a search under way for the mRNAs for the storage proteins of legume cotyledons and the larger and smaller subunits of the fraction I protein.

\section{Nuclear magnetic order in solid ${ }^{3} \mathrm{He}$}

from a Correspondent

THE long predicted nuclear magnetic ordering transition in solid ${ }^{3} \mathrm{He}$ seems to have been observed at a temperature of $1.17 \mathrm{mK}$ in an experiment carried out at Cornell University by Halperin, Archie, Rasmussen, Burhman and Richardson, and reported in a recent issue of Physical Review Letters (32, 927; 1974).

The compressional cooling technique used in this work, which has been developed during the past few years by the low temperature group at Cornell, has already been extraordinarily fruitful in that it has led to discovery of the two new and apparently superfluid states of liquid ${ }^{3} \mathrm{He}$. The technique, known as Pomeranchuk cooling after the Russian physicist who originally suggested it, is based on the fact that for temperatures below about $0.3 \mathrm{~K},{ }^{3} \mathrm{He}$ has a negative latent heat of solidification so that, unlike other materials, it absorbs heat while changing phase from liquid to solid.

In practice, after being precooled to about $15 \mathrm{mK}$ by means of a dilution refrigerator, the liquid is then steadily compressed by the application of external pressure. At a pressure of about 34 atmospheres it starts to solidify and, because heat is thus being absorbed, it also starts to cool. The cooling effect can be maintained until, eventually, the cell is entirely filled with solid. The final temperature depends on a number of factors including the starting temperature, the rate at which heat leaks into the system or is developed by mechanical inefficiencies in the compression mechanism itself, and the rate at which the liquid is compressed, but is usually in the range $1-2 \mathrm{mK}$. The ${ }^{3} \mathrm{He}$, which is the subject of the present experiments, is thus able to act as its own refrigerant from which accrues the enormous benefit that the usual formidable problems of thermal transfer and equilibrium in the low $\mathrm{mK}$ temperature range are automatically minimised.

Unlike ${ }^{4} \mathrm{He}$, the ${ }^{3} \mathrm{He}$ nucleus possesses a magnetic dipole moment and it is to be expected that, at some sufficiently low temperature $T_{\mathrm{c}}$, a transition will occur in which the nuclei become aligned in some ordered fashion relative to each other. Because the nuclear moments are so tiny the ordering temperature is bound to be exceedingly small and, just considering the direct magnetic interaction of the nuclei, it may be calculated that $T_{\mathrm{c}} \sim 10^{-6} \mathrm{~K}$. More refined calculations, however, taking account of the so-called exchange forces, suggest $T_{\mathrm{c}} \sim 10^{-3} \mathrm{~K}$, which is just within the experimental range made accessible by Pomeranchuk cooling.

In essence the basic Cornell experiment was extremely simple: the volume occupied by the ${ }^{3} \mathrm{He}$ was reduced at a steady rate while its pressure was continuously monitored. The pressure, with a mixture of liquid and solid in the cell, was necessarily always that of the melting curve at the relevant temperature and, since the melting pressure increases with falling temperature, the pressure automatically gave a measure of the temperature. Under these conditions a plot of pressure $P$ against $t$ is almost linear, corresponding to cooling of the sample at a constant rate, provided that no phase changes occurred in either liquid or solid apart, of course, from the continuous solidification-action process responsible for the refrigeration. In practice, the original experiment showed one change of slope and one discontinuity in the $P(t)$ plot, and these were eventually proved to be caused by two superfluid transitions in the liquid phase. In the present work a third anomaly in the $P(t)$ graph was observed at the even lower temperature of $\mathbf{1 . 1 7}$ mK.

The interpretation of these experiments, where both liquid and solid are simultaneously present, is of course extremely difficult. Through a series of ingenious additional experiments, however, in which heat pulses were applied to the helium while compression was continued at a steady rate, the authors seem to have demonstrated unambiguously that the $P(t)$ anomaly at $1.17 \mathrm{mK}$ corresponds to a phase change within the solid, which must presumably represent the onset of magnetic ordering, and they have been able to deduce that the entropy of the solid falls by a factor of 5 within a temperature range of only $100 \mu \mathrm{K}$.

It has not been possible from these experiments to deduce the nature of the magnetic order below $T_{\text {c. }}$. Measurements of the nuclear susceptibility at higher temperatures had led to the development of a detailed model which predicted a transition at $2.2 \mathrm{mK}$ to an antiferromagnetic state, in which neighbouring nuclei point in opposite directions. The fact that the actual transition has now been found to occur at only half this temperature is in itself strong evidence of the inadequacy of this model. A great deal of further work will certainly be required before a proper understanding of magnetic ordering in solid ${ }^{3} \mathrm{He}$ can be attained, but it is very encouraging to know that the phenomenon occurs in a temperature range which has now become accessible to experiment.

\section{Chemical heat at oceanic ridges}

from Peter J. Smith

Geomagnetism Correspondent

NEAR the crests of active oceanic ridges the mean heat flow is generally higher than the global average, and measurements are considerably more scattered than those in, for example, typical ocean basins. Both of these observations are explicable in general terms, for hotter mantle-derived material rises beneath ridge crests to form new ocean floor, and a combination of rugged ridge topography and uneven sediment cover tends to produce both high measurement errors and a distortion of the heat flow pattern (for example, by thermal refraction). Nevertheless, in view of the importance of testing the seafloor spreading hypothesis as rigorously as possible, such loose explanations are not entirely satisfactory; and it is not unreasonable to suspect that the rather messy heat flow picture may be concealing important information about physical and chemical processes in mid-ocean regions.

Because of the large scatter of data, for example, there may be legitimate doubt about the validity of the mean heat flow values themselves, all the more so as McKenzie ( $J$. geophys. Res., 72,6261 ; 1967) has shown that they are inconsistent with the formation of new oceanic crust at a reasonable temperature. Mean heat flow as measured over ridge crests may indeed be high, but it is not as high as might be reasonably expected.

Recognising the scope for investigation here, Lister (Geophys. J., 26, 515 ; 1972) made an important series of heat flow measurements on the Juan de Fuca ridge and near the Explorer trough in which he took care to assess topographic effects and the thermal influence of recent sediment disturbances. The conclusion he came to was that the thermal balance of a ridge crest zone can be interpreted in terms of a 